\title{
Peritoneal Gastrointestinal Stromal Tumor
}

National Cancer Institute

\section{Source}

National Cancer Institute. Peritoneal Gastrointestinal Stromal Tumor. NCI Thesaurus.

Code C40338.

A gastrointestinal stromal tumor that arises from the peritoneum. This is an extragastrointestinal tumor, meaning that it does not arise directly from the gastrointestinal tract. 